
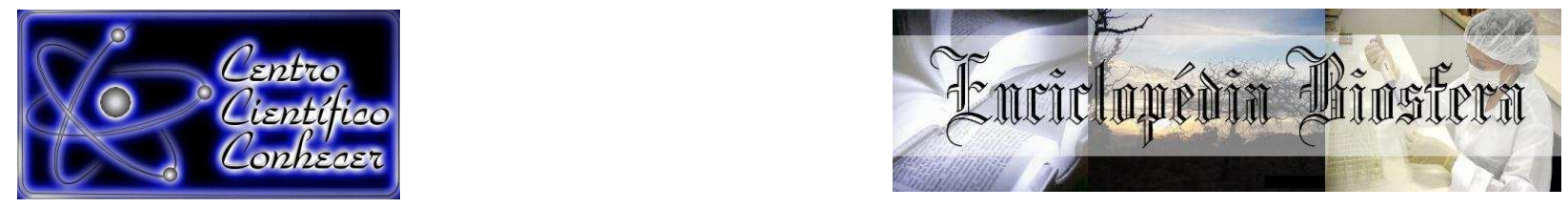

\title{
BIOINDICADORES DE QUALIDADE EDÁFICA EM DIFERENTES USOS DO SOLO
}

Diego Armando Amaro da Silva ${ }^{1}$, Danni Maisa da Silva ${ }^{2}$, Rodrigo Josemar Seminoti Jacques $^{3}$, Zaida Inês Antoniolli ${ }^{3}$

1 Eng ${ }^{\circ}$ Agro Mestrando do Programa de Pós-graduação em Agronomia - Agricultura e Ambiente da Universidade Federal de Santa Maria, Bolsista Capes

(damaro.agro@gmail.com), Frederico Westphalen, RS, Brasil.

2 Professora Doutora da Universidade Estadual do Rio Grande do Sul, Três Passos, Brasil.

3 Professor(a) Doutor(a), Universidade Federal de Santa Maria, Santa Maria, Brasil.

Recebido em: 08/09/2015 - Aprovado em: 14/11/2015 - Publicado em: 01/12/2015

DOI: http://dx.doi.org/10.18677/Enciclopedia_Biosfera_2015_260

Nas áreas de encosta do Rio Grande do Sul a vulnerabilidade à degradação do solo é elevada devido à combinação de relevo fortemente ondulado a montanhoso e solos com pequena profundidade efetiva. Desta forma, o manejo do solo onde os usos do solo podem levar desde um sistema sustentável até mesmo a um cenário de degradação. Objetivou-se avaliar a influência de diferentes usos do solo sobre a fauna edáfica e indicar os melhores bioindicadores de qualidade do solo nos ambientes testados. O trabalho foi conduzido no município de Silveira Martins, RS, em delineamento inteiramente casualizado com cinco usos do solo (campo nativo, florestamento de eucalipto, pomar de pessegueiros, bananeiras, e citros) com quatro repetições cada. Foram usadas armadilhas do tipo PROVID, que permaneceram sete dias no campo. Os grupos taxonômicos mais abundantes foram: Collembola e Hymenoptera, sendo que o campo nativo apresentou maior abundância, e a maioria dos tratamentos apresentam dominância destas ordens. O pomar de pessegueiros possui maior diversidade de organismos. A Análise de Componentes Principais (ACP) é capaz de explicar $92 \%$ da variância até o segundo componente, sendo valiosa para discriminar os atributos indicadores de qualidade do solo. Os grupos Coleoptera, Hemiptera, Diptera, Hymenoptera e Isopoda podem ser utilizados como bioindicadores de qualidade edáfica nos usos do solo testados no Rebordo do Planalto Gaúcho.

PALAVRAS-CHAVE: densidade, diversidade, qualidade do solo, sustentabilidade.

\section{EDAPHIC QUALITY BIOINDICATORS IN DIFFERENT USES OF SOIL}

\begin{abstract}
In the hillside areas of RS state, the vulnerability to land degradation is high due to the combination of strongly wavy relief and the mountainous soils with little effective depth, where land uses can take anywhere from a sustainable system even to a degradation scenario. The objective of this study was to evaluate the influence of different land uses on soil fauna and indicate the best soil quality bio-indicators in the
\end{abstract}


tested environments. The work was conducted in the city of Silveira Martins, RS, in a completely randomized design with five land uses (native grass, eucalyptus forestry, orchard peach, orchard banana and orchard citrus) with four replications. Were used PROVID traps, which remained seven days in the field. The most abundant taxa were: Collembola and Hymenoptera, with native grass being more abundant, and most treatments show dominance of these orders. The peach orchard has greater diversity of organisms. The Principal Component Analysis is able to explain $92 \%$ of the variance to the second component, being valuable for discriminating attributes of soil quality indicators. The PCA indicated that the groups Coleoptera, Hemiptera, Diptera, Hymenoptera and Isopoda can be used as bio-indicators of the soil quality in land use tested in the region of Plateau Border in Rio Grande do Sul state.

KEYWORDS: density, diversity, soil quality, sustainability.

\section{INTRODUÇÃO}

A região do Rebordo do Planalto do Rio Grande do Sul apresenta relevo predominantemente acidentado, podendo variar de suave ondulado a montanhoso, sendo caracterizada como áreas de encosta (MIGUEL et al., 2011), com solos pouco desenvolvidos e frágeis. Nessas áreas podem ser encontrados diversos usos da terra relacionados à agricultura, pastagem plantada, áreas de floresta, áreas de pousio e campo nativo, as quais apresentam diferentes graus de suscetibilidade e degradação ambiental (PEDRON et al., 2009; SAMUEL-ROSA et al., 2011; MIGUEL et al., 2011).

A base produtiva desta região consiste na agricultura familiar, a qual se caracteriza por sua baixa rentabilidade e caráter de subsistência (NEUMANN et al., 2007). As atividades com maior aptidão ao relevo declivoso correspondem a horticultura, fruticultura, produção avícola e agricultura (feijão, milho e fumo) que podem levar a degradação destes solos, que são altamente vulneráveis aos impactos antrópicos e de grande vulnerabilidade, devido à associação de relevo altamente declivoso e solos com pequena profundidade efetiva (ZALAMENA, 2008).

Os biomas nativos brasileiros possuem uma das maiores biodiversidades do planeta (SILVA et al., 2012). Porém a substituição de sistemas nativos por sistemas agrícolas, resulta em modificações na composição e diversidade dos grupos mais frequentes de organismos edáficos em diferentes graus de intensidade (NUNES et al., 2012), seja por meio de mudanças nas práticas de manejos (ALMEIDA et al., 2013) associadas ao sistema de cultivo, adubação, calagem, ou até mesmo a aplicação de agrotóxicos (DESCHER et al., 2011).

Entretanto, essa comunidade de invertebrados pode ser conservada quando o sistema de manejo do solo derivado tem uma estrutura ambiental similar ao sistema de origem (NUNES et al., 2012), ou seja, estes organismos servem como bioindicadores da qualidade do solo e do ambiente em questão (BARETTA et al., 2011). Assim, uma das maneiras de se avaliar o impacto das práticas de manejo no funcionamento dos ecossistemas é por meio do monitoramento da abundância e diversidade dos organismos do solo.

Desta forma, objetivou-se avaliar a influência de diferentes usos do solo no Rebordo do Planalto Gaúcho sobre a abundância e diversidade de grupos da comunidade edáfica, além de indicar os melhores bioindicadores de qualidade do solo nos ambientes testados. 


\section{MATERIAL E MÉTODOS}

O estudo foi realizado em propriedade rural no município de Silveira Martins RS (53\%3'45"W e 290'24"S), com altitude média de $450 \mathrm{~m}$. Segundo a classificação climática de Köppen, o clima da região é Cfa ou subtropical úmido, com precipitação média anual entre 1500 e $1750 \mathrm{~mm}$, sendo os meses de julho, agosto e setembro a época de concentração máxima de precipitação, enquanto novembro, dezembro e março são os meses que apresentam menor índice pluviométrico. A temperatura anual situa-se entre 18 e $20^{\circ} \mathrm{C}$ (MORENO, 1961).

O solo da região do Rebordo do Planalto Gaúcho caracteriza-se pela associação entre Neossolos e Chernossolos (EMBRAPA, 2013), conhecido como associação Ciríaco-Charrua, cujas características físicas e químicas de $0-10 \mathrm{~cm}$ de profundidade se encontram na Tabela 1. O experimento constou de cinco tratamentos: campo nativo, florestamento de eucalipto, pomar de pessegueiros, pomar de bananeiras e pomar de citros.

TABELA 1. Composição e características químicas e físicas das cinco áreas analisadas.

\begin{tabular}{|c|c|c|c|c|c|c|}
\hline Análise & Unidade & $\mathbf{C N}^{1}$ & EUC & PES & BAN & CIT \\
\hline pH em água & $-\log \left[\mathrm{H}^{+}\right]$ & 5,6 & 5,8 & 5,5 & 5,7 & 5,7 \\
\hline Argila & $\mathrm{g} \mathrm{kg}^{-1}$ & 37,6 & 32,4 & 36,1 & 29,5 & 34,2 \\
\hline Matéria orgânica (MOS) & $\mathrm{g} \mathrm{kg}^{-1}$ & 2,4 & 3,4 & 3,2 & 2,9 & 4,1 \\
\hline $\mathrm{P}\left(\right.$ Mehlich $\left.^{-1}\right)$ & $\mathrm{mg} \mathrm{dm} \mathrm{m}^{-3}$ & 5,5 & 6,8 & 6,7 & 7,3 & 6,3 \\
\hline $\mathrm{K}^{+}$ & $\mathrm{mg} \mathrm{dm} \mathrm{m}^{-3}$ & 73 & 71 & 65 & 64 & 88 \\
\hline $\mathrm{Al}^{3+}$ & $\mathrm{cmol}_{\mathrm{c}} \mathrm{dm}^{-3}$ & 0,0 & 0,0 & 0,1 & 0,1 & 0,0 \\
\hline $\mathrm{Ca}^{++}$ & $\mathrm{cmol}_{\mathrm{C}} \mathrm{dm}^{-3}$ & 1,8 & 2,9 & 2,1 & 2,4 & 3,2 \\
\hline $\mathrm{Mg}^{++}$ & $\mathrm{cmol}_{\mathrm{c}} \mathrm{dm}^{-3}$ & 1,4 & 0,9 & 1,0 & 1,1 & 1,2 \\
\hline Capacidade de Troca de Cátions (СТC) & $\mathrm{cmol}_{\mathrm{C}} \mathrm{dm}^{-3}$ & 4,6 & 4,9 & 5,1 & 5,2 & 5,8 \\
\hline Saturação da CTC por bases (V\%) & $\%$ & 91,3 & 93,8 & 80,3 & 80,8 & 89,5 \\
\hline
\end{tabular}

${ }^{1} \mathrm{CN}=$ Campo nativo; EUC = Florestamento de eucalipto; PES = Pomar de pessegueiros; $\mathrm{BAN}=$ Pomar de bananeiras; $\mathrm{CIT}=$ Pomar de citros.

O campo nativo é estabelecido e não sofreu manejo algum nos últimos 30 anos, tanto na pastagem quanto no solo. O florestamento de eucalipto (Eucalyptus grandis) foi implantado em 2006, estando com cinco anos durante as coletas. $\mathrm{O}$ pomar de pessegueiros possui cerca de 20 plantas, com espaçamento de $4 \times 2 \mathrm{~m}$, logo ao lado do pomar de bananeiras, que possui cerca de 40 plantas sem espaçamento pré-definido na implantação. Ambas foram plantadas em 2003, oito anos antes das coletas. O pomar de citros foi estabelecido no final da década de 80 de acordo com o proprietário. Todos os pomares implantados, assim como o florestamento de eucalipto não possuíram adubação de base na ocasião da implantação.

A avaliação da fauna do solo foi realizada pelo método PROVID (ANTONIOLLI, 2006), onde a armadilha foi constituída por uma garrafa pet de 2 I, com janelas laterais de $6 \times 4 \mathrm{~cm}$ à $20 \mathrm{~cm}$ de sua base. No interior da garrafa foram adicionados $200 \mathrm{ml}$ de solução $70 \%$ álcool $+25 \%$ água $+5 \%$ glicerina.

Foram instaladas quatro armadilhas por tratamento em janeiro de 2011, e permaneceram no campo durante sete dias de modo que os bordos das aberturas 
das armadilhas ficassem ao nível do solo. Após a retirada das armadilhas do campo, estas foram encaminhadas ao Laboratório de Biologia e Microbiologia do Solo e Ambiente da Universidade Federal de Santa Maria. As amostras foram passadas em peneiras de 10 e 100 Mesh, para separar o solo e os resíduos vegetais da fauna coletada, e em seguida foi efetuada a identificação dos organismos ao nível de ordem, com auxílio de lupa binocular.

A fauna foi avaliada quantitativamente por meio da abundância das ordens, e qualitativamente por meio do Índice de Dominância de Simpson (Is) e do Índice de Diversidade de Shannon $(H)$, usando o software BioDap ${ }^{\circledR}$. O Is expressa à probabilidade de indivíduos de uma mesma comunidade pertencerem a mesma espécie, variando de 0 a 1, quanto mais alto sendo o valor, maior a dominância de uma espécie sobre as demais, e é definido como: : Is $=\mathrm{N}(\mathrm{N}-1) / \mathrm{ni}(\mathrm{ni}-1)$, onde: $\mathrm{ni}=$ densidade de cada ordem; $\mathrm{N}=$ número total de indivíduos do grupo. $\mathrm{O} \mathrm{H}$ reflete a incerteza de apanhar ao acaso um indivíduo da mesma espécie, e pode variar de 0 a 5, havendo maior diversidade de organismos, quanto mais alto o índice, que é definido como: $\mathrm{H}=-\Sigma$ (pi log pi), onde: $\mathrm{pi}=\mathrm{ni} / \mathrm{N} ; \mathrm{ni}=$ densidade de cada ordem; $\mathrm{N}=$ número total de indivíduos do grupo.

Os dados obtidos foram submetidos a estatística descritiva e análise multivariada por meio da técnica de Análise de Componentes Principais.

\section{RESULTADOS E DISCUSSÃO}

O uso do solo afetou a distribuição da fauna edáfica, onde foram coletados um total de 11 ordens diferentes subdivididos em quatro classes: Entognatha $(54,6 \%$ dos indivíduos), Insecta (35,9\%), representada por sete ordens, Arachnida $(9,4 \%)$, representada por duas ordens, e Malacostraca (0,2\%) (Tabela 2).

TABELA 2. Frequência da fauna edáfica, Índice de Diversidade de Shannon $(H)$, Índice de Dominância de Simpson (Is) sob cinco usos do solo no Rebordo do Planalto Gaúcho.

\begin{tabular}{ccccccccccccccccc}
\hline \multirow{2}{*}{ Trat. ${ }^{1}$} & \multicolumn{10}{c}{ Ordem } & \multicolumn{1}{c}{ Abundância } & H & Is & Riqueza \\
\cline { 2 - 6 } & AC & AR & CL & CP & DE & DI & HE & HY & IP & IS & OR & & & & & \\
\hline Campo nativo & 23 & 8 & 184 & 19 & 4 & 28 & 5 & 167 & - & 28 & 3 & 467 & 1,54 & 0,29 & 11 \\
Eucaliptos & 50 & 7 & 147 & 8 & 1 & 13 & 2 & 40 & 3 & 11 & 1 & 282 & 1,51 & 0,32 & 10 \\
Pessegueiros & 11 & 2 & 41 & 3 & 1 & 12 & 7 & 38 & - & 4 & 1 & 118 & 1,70 & 0,24 & 10 \\
Bananeiras & 9 & 2 & 105 & 12 & - & 7 & 1 & 19 & - & 9 & - & 163 & 1,26 & 0,43 & 8 \\
Citros & 15 & 13 & 326 & 17 & - & 9 & 1 & 55 & - & 3 & 5 & 443 & 1,01 & 0,56 & 9 \\
\hline
\end{tabular}

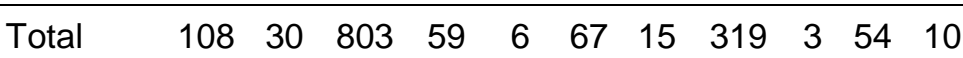

${ }^{1}$ Tratamentos. ${ }^{2} \mathrm{AC}=$ Acari; $\mathrm{AR}=$ Aranae $; \mathrm{CL}=$ Collembola $\mathrm{CP}=$ Coleoptera; $\mathrm{DE}=$ Dermaptera; $\mathrm{DI}=$ Diptera; HE = Hemiptera; HY = Hymenoptera; IP = Isopoda; IS = Isoptera; OR = Orthoptera.

O campo nativo apresentou o maior número de organismos coletados (467 indivíduos), seguido pelo pomar de citros, florestamento de eucalipto, pomar de bananeiras e, por último, o tratamento que apresentou menor abundância de organismos, o pomar de pessegueiro. 
Como resultado, ao contrário do observado por ALMEIDA et al. (2013) esperava-se que o tratamento campo nativo apresentasse um número total de indivíduos menor que os coletados nos demais tratamentos, mas o elevado número de organismos das ordens Collembola e Hymenoptera fez com que este tratamento fosse o de maior abundância. Esta dominância foi detectada em todos os tratamentos, correspondendo a $54,6 \%$ e $21,6 \%$ dos organismos coletados no experimento, respectivamente, concordando com os resultados encontrados por SANTOS et al. (2013).

A dominância causada pela ordem Hymenoptera, se deve provavelmente pela proximidade das áreas, uma vez que as formigas possuem elevada mobilidade, transitando de uma área para outra com facilidade (ARAÚJO et al., 2010). Porém, LAVELLE \& SPAIN (2001) afirmam que não se deve relacionar a alta abundância desta ordem somente com prejuízos à agricultura, pois a maior parte dos grupos de formigas não são desfolhadores, sendo consideradas de fundamental importância para a manutenção da qualidade do solo, sendo úteis como bioindicadores dessas condições (CREPALDI et al., 2014).

VASCONCELLOS et al. (2015) destacaram em trabalho analisando o impacto de áreas agrícolas sobre o habitat e desenvolvimento da ordem Isopoda, que áreas de mata podem constituir um importante refúgio para este grupo de organismos, corroborando com o encontrado no presente trabalho, visto que a ordem foi encontrada em apenas um dos locais, justamente o florestamento de eucalipto analisado.

A riqueza é uma das variáveis que é levada em consideração no Índice de Shannon $(H)$. Sendo assim, é natural que tratamentos com maior riqueza de espécies apresentem um valor maior. O florestamento de eucalipto apresentou maior riqueza de organismos com um total de 11 ordens, seguido do campo nativo e pomar de pessegueiros (dez ordens), o pomar de citros (nove ordens), e pomar de bananeiras, com apenas oito ordens.

O florestamento de eucalipto e o campo nativo apresentaram valores similares entre si para os dois índices, e assim como o pomar de bananeiras, apresentaram a ordem Collembola como dominantes. Porém de forma menos acentuada quando comparada ao pomar de citros, refletindo diretamente sobre os índices analisados (maior Is e menor $\mathrm{H}$ ), mostrando ser o tratamento com maior dominância de uma ordem (Collembola) sobre as demais.

O local com menor dominância (Is), resultando em uma diversidade maior $(\mathrm{H})$ foi o pomar de pessegueiros, tratamento no qual as ordens Collembola e Hymenoptera, predominantes no experimento, apresentaram menor número de indivíduos. O pomar de pessegueiros demonstrou ter o maior valor para $\mathrm{H}$, provavelmente devido ao baixo número de organismos totais (119 indivíduos) quando comparado aos demais tratamentos, juntamente com um número elevado de ordens presentes.

Os dados ainda foram submetidos à Análise dos Componentes Principais (ACP). A ACP é uma das técnicas da estatística multivariada que são consideradas como importantes ferramentas no estudo de indicadores de qualidade do solo (BARETTA, 2007), e procura explicar a estrutura da variância-covariância por meio de combinações não correlacionadas com as variáveis originais, sendo que cada componente é independente (RIBAS \& VIEIRA, 2011). Os resultados obtidos encontram-se na Tabela 3, e a representação gráfica da ACP na Figura 1. 
TABELA 3. Pesos dos atributos analisados sobre os componentes principais.

\begin{tabular}{|c|c|c|c|c|c|}
\hline \multirow{2}{*}{ Variável $^{1}$} & \multicolumn{2}{|c|}{ Componente principal } & \multirow{2}{*}{ Variável $^{2}$} & \multicolumn{2}{|c|}{ Componente principal } \\
\hline & 1 & 2 & & 3 & 4 \\
\hline$A C$ & $-0,684$ & 0,669 & MS & $\underline{0,923}$ & 0,385 \\
\hline$A R$ & $-0,639$ & 0,693 & MOS & $\underline{0,976}$ & 0,207 \\
\hline$C L$ & $-0,584$ & 0,728 & Arg & $\underline{-0,978}$ & $-0,206$ \\
\hline $\mathrm{CP}$ & $-0,980$ & $-0,192$ & $\mathrm{pH}$ & 0,380 & 0,762 \\
\hline DE & $\underline{-0,976}$ & $-0,207$ & CTC & $\underline{0,976}$ & 0,207 \\
\hline DI & $\underline{-0,983}$ & $-0,177$ & V\% & $-0,613$ & 0,749 \\
\hline $\mathrm{HE}$ & $-0,253$ & $-0,651$ & $\mathrm{Al}$ & 0,592 & $\underline{-0,804}$ \\
\hline HY & $\underline{-0,998}$ & $-0,068$ & $\mathrm{Ca}$ & $\underline{0,963}$ & 0,266 \\
\hline IP & 0,390 & 0,695 & $\mathrm{Mg}$ & $-0,969$ & $-0,241$ \\
\hline IS & $\underline{-0,976}$ & $-0,207$ & $P$ & $\underline{0,985}$ & 0,172 \\
\hline OR & $-0,639$ & 0,693 & K & $-0,612$ & 0,779 \\
\hline
\end{tabular}

${ }^{1}$ Atributos biológicos na primeira coluna; ${ }^{2}$ Massa seca da serrapilheira/mantilho e atributos físicos e químicos do solo na segunda coluna.

As variáveis foram distribuídas em dois componentes principais que explicaram juntas $92,26 \%$ da variância detectada. O primeiro componente explicou $65,76 \%$ da variância total, sendo composto pela massa seca da serrapilheira (MS), MOS, $\mathrm{Ca}$, e P, além de apresentar forte relação negativa com as ordens $\mathrm{CP}, \mathrm{HE}, \mathrm{DI}$, HY e IS, além do teor de argila e Mg presente nas áreas. Estas variáveis, de acordo com a ACP, foram importantes para diferenciar adequadamente as áreas de estudo, servindo como indicadores de qualidade do solo, a exemplo do que pode ser observado na Figura 1, onde os atributos MOS, Ca e P ficam visivelmente distantes do ambiente campo nativo, porém muito próximo dos atributos $\mathrm{Mg}, \mathrm{CP}, \mathrm{DE}, \mathrm{DI}$, e HY, que caracterizaram esta área, como pode ser visto também na Tabela 1.

Já o segundo componente explicou $26,52 \%$ da variância total, e foi composto pelo atributo Al, que devido à ausência nos demais tratamentos, caracterizou-se por ser observado nos pomares de bananeiras e pessegueiros. 


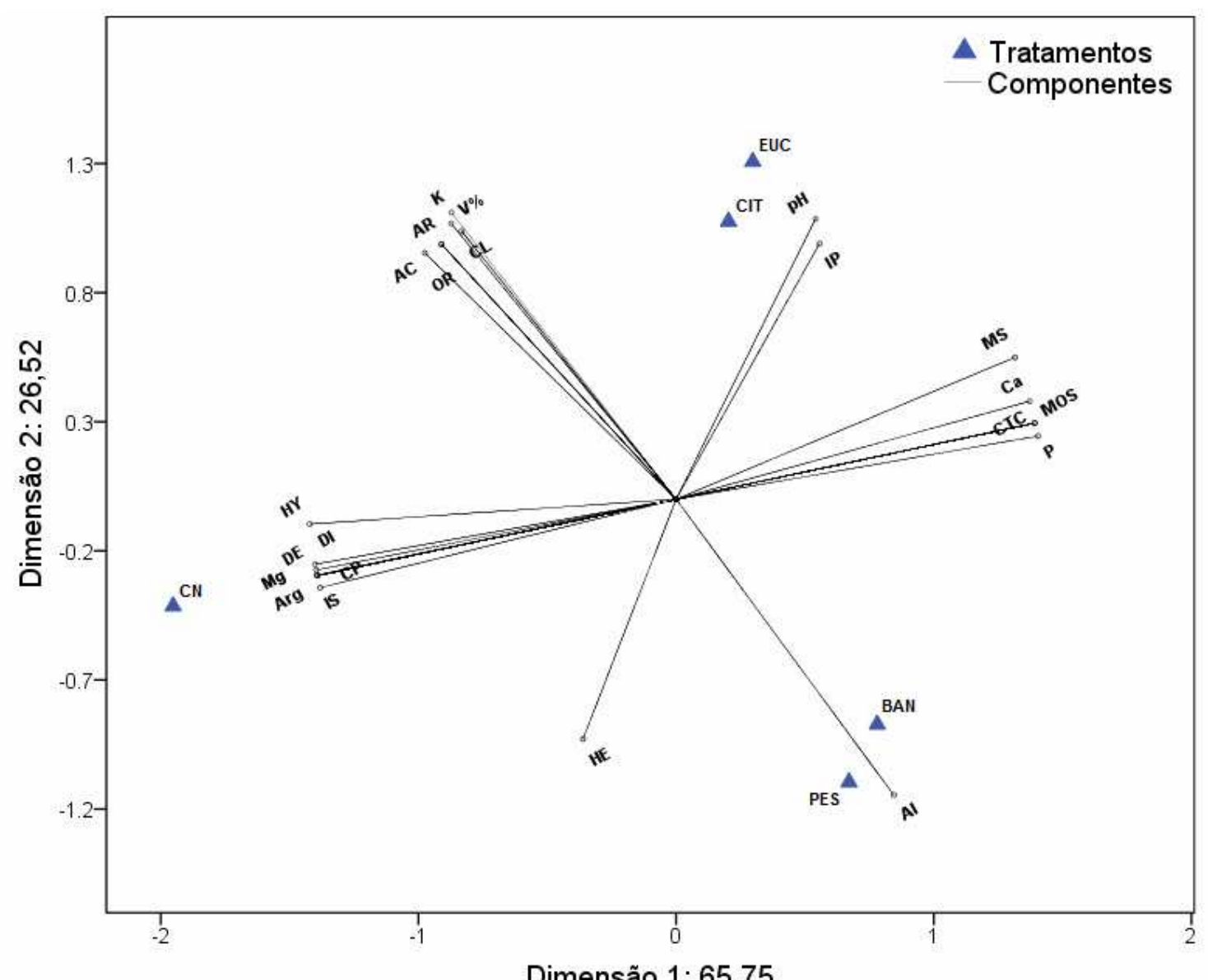

FIGURA 1 Representação gráfica da Análise de Componentes Principais entre as áreas testadas, a massa seca da serrapilheira coletada (MS) e os atributos físicos, químicos e biológicos do solo. Fonte: Autores. ${ }^{*} \mathrm{CN}=$ Campo nativo; EUC = Florestamento de eucalipto; PES = Pomar de pessegueiros; $\mathrm{BAN}=$ Pomar de bananeiras; $\mathrm{CIT}=$ Pomar de citros.

Nesse estudo, a ACP indicou que os tratamentos que sofreram algum tipo de intervenção antrópica, seja por processo de construção e recuperação do solo ou por ocasião de desmatamento e transformação em áreas agrícolas, estão muito próximos entre si e separados do ambiente nativo observado, o qual não apresenta intervenção do homem (OLIVEIRA FILHO et al., 2015). Ainda, FERREIRA et al. (2013) afirmam que o manejo do solo deve constar de práticas que apresentem um embasamento sustentável, pois um solo bem manejado, na qual pode-se encontrar uma diversidade de seres vivos de diferentes reinos, é fundamental para uma satisfatória produção agrícola, gerando assim um equilíbrio entre a produção agrícola e a biota do solo.

\section{CONCLUSÕES}

A fauna edáfica é afetada pelos distintos usos do solo na região do Rebordo do Planalto Gaúcho. As ordens taxonômicas Collembola e Hymenoptera apresentam dominância em todos os locais analisados. O campo nativo demonstra elevada abundância, enquanto o pomar de pessegueiros proporciona maior diversidade de 
organismos, porém com menor abundância de indivíduos. Os pomares de citros e bananeiras apresentam baixa diversidade de organismos na região de estudo, devido principalmente ao elevado número de exemplares da ordem Collembola.

Os grupos taxonômicos Coleoptera, Hemiptera, Diptera, Hymenoptera e Isopoda são os melhores bioindicadores de qualidade edáfica nos diferentes usos do solo testados na região do Rebordo do Planalto Gaúcho.

\section{REFERÊNCIAS}

ALMEIDA, N.S. et al. Efeito do preparo do solo na fauna epiedáfica em cultivo de Nogueira-pecã. Cadernos de Agroecologia, v.8, n.2, p.1-5, 2013.

ANTONIOLLI, Z.I. et al. Método alternativo para estudar a fauna do solo. Ciência Florestal, v.16, n.4, p.407-417, 2006.

ARAÚJO, L.H.A. et al. Macrofauna edáfica sob diferentes ambientes em latossolo da região agreste. In: IV Congresso Brasileiro de Mamona e I Simpósio Internacional de Oleaginosas Energéticas. João Pessoa. Anais... João Pessoa: CBM, 2010.

BARETTA, D. et al. Fauna edáfica e qualidade do solo. Tópicos em Ciência do Solo, v.7, n.1, p.119-170, 2011.

BARETTA, D. Fauna do solo e outros atributos edáficos como indicadores da qualidade ambiental em áreas com Araucaria angustifólia no Estado de São Paulo. São Paulo, 2007. 158p. Tese (Doutorado em Agronomia) - Escola Superior de Agricultura "Luiz de Queiroz", Piracicaba, 2007.

CREPALDI, R.A. et al. Formigas como bioindicadores da qualidade do solo em sistema integrado lavoura-pecuária. Ciência Rural, v.44, n.5, p.781-787, 2014.

DRESCHER, M.S. et al. Fauna epigeica em sistemas de produção de nicotiana tabacum L. Revista Brasileira de Ciência do Solo, v.35, n.5, p.1499-1507, 2011.

EMBRAPA, Empresa Brasileira de Pesquisa Agropecuária. Sistema brasileiro de classificação de solos. 3 ed. Brasília, 2013. 353 p.

FERREIRA, T.C. et al. População de Pherethima hawayana sob diferentes tipos de manejo do solo. Revista Eletrônica de Biologia, v.6, n.1, p.24-34, 2013.

LAVELLE, P; SPAIN, A.. Soil Ecology. Amsterdam: Kluwer Scientific Publications, 2001. 654p.

MIGUEL, P. et al. Solo e dinâmica de ocupação das terras em áreas do rebordo do planalto do Rio Grande do Sul. Revista Brasileira de Agrociência, v.17, n.4, p.447455, 2011.

MORENO, J.A. Clima do Rio Grande do Sul. Porto Alegre: Secretaria da Agricultura e Abastecimento, Diretoria de Terras e Colonização, Secção de Geografia, 1961. 
NEUMANN, P.S. et al. Agroindústria familiar de massas na região da quarta colônia do Rio Grande do Sul. In: Congresso Sociedade Brasileira de Economia, Administração e Sociologia Rural, 45., 2007, Londrina, Anais... Londrina: SOBER, 2007.

NUNES, L.A.P.L. et al. Caracterização da fauna edáfica em sistemas de manejo para produção de forragens no Estado do Piauí. Revista Ciência Agronômica, v.43, n.1, p.30-37, 2012.

OLIVEIRA FILHO, L.C.I. et al. Mesofauna de solo construído em área de mineração de carvão. Revista de Ciências Agroveterinárias, v.14, n.1, p.55-64, 2015.

PEDRON, F.A. et al. et al. Morfologia e Classificação Taxonômica de Neossolos e Saprolitos derivados de rochas vulcânicas da Formação Serra Geral no Rio Grande do Sul. Revista Brasileira de Ciência do Solo, v. 33, n.1, p. 119-128, 2009.

SAMUEL-ROSA, A. et al. Uso da terra no Rebordo do Planalto do Rio Grande do Sul. Ciência e Natura, v. 33, n. 1, p.161-173, 2011.

SANTOS, C.C. et al. Fauna invertebrada epigeica associada ao cultivo de café em sistema agroecológico no Território do Vale do Ivinhema, Mato Grosso do Sul. Cadernos de Agroecologia, v.8, n.2, p.1-5, 2013.

SILVA J. et al. Fauna do solo em sistemas de manejo com café. Journal of Biotechnology and Biodiversity, v.3, n.2, p.59-71, 2012.

VASCONCELLOS, N.J.S. et al. Reservas legais: um importante refúgio para os isópodas terrestres em áreas agrícolas. Revista Ambiente \& Água, v.10, n.3, p.676684, 2015.

ZALAMENA, J. Impacto do uso da terra nos atributos químicos e físicos de solos do Rebordo do Planalto - RS. 2008. Dissertação (Mestrado em Ciência do Solo) - Universidade Federal de Santa Maria, Santa Maria, RS, 2008. $79 \mathrm{f}$. 